\title{
Great Success Can Lie Ahead
}

A Special Plenary Session on Science and Society took place during the 15th General Conference of the Condensed Matter Division of EPS (Baveno-Stresa; 22-25 April 1996). The highlight was a presentation by J.C. Phillips of Lucent Technologies, Murray Hill, NJ, USA, entitled Past and Future of Academic and Industrial Research in Solid-State Physics which led to a lively discussion. The following text, which is accompanied by comments by J.T. Devreese and J.Bessa Sousa, is based on his presentation and the paper submitted to the conference proceedings (to be published in the autumn by Physica Scripta).

\section{The increasing focus on near-term results by research in industry owing to highly competitive world markets has drastic implications. Physics in general and solid-state physics in particular, must react by stimulating small science, looking beyond fundamental understanding and promot- ing disciplined academic-industrial-governmental interactions.}

Many laboratories are undergoing significant changes owing to two main factors. They are the growing population of cheap and easily trained skilled labour, produced by what many call overpopulation, and the emergence of world markets and world-wide industrialization brought about by networked computers. These revolutionary factors are affecting certain "high technology" businesses much more than others, but all industrialized activity will be strongly influenced.

Many economists are convinced that surplus value leading to wealth generation is produced by manufacturing and not by the so-called service or information economies. The western world's problem is that products based on many of its discoveries are now being manufactured more profitably in the Far East. Moreover, modern information technology means that others know as much about markets as we do.

Structural change means that many conflicts are inherent in the emerging worlds of science and technology. While it may be difficult to resolve them completely, physics can develop approaches that encourage one to believe that many great successes should still lie ahead.

\section{Emphasize Small Science}

There has been much debate on how physics should respond to the recent changes. My first thesis is that, whether the technology is high, medium of low, the best preparation for physicists is provided by research in small science. This point has been hotly debated in the United

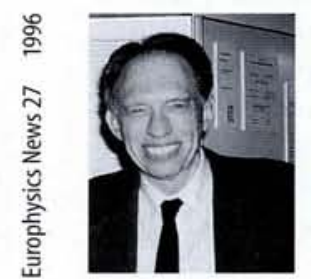

J.C. Phillips, formerly Director of Research at the AT\&T Bell Laboratories, Murray Hill, is presently with Lucent Technologies, 5000 Mountain Ave., Murray Hill, NJ 07974, USA, the former systems and technology divisions of AT\&T plus Bell Labs.
States. It seemed that large science, in the form of the Superconducting Super Collider (SSC), was destined to destroy small science. As time passed, the budget for the SSC ballooned from 6000 to 12000 million dollars, and it was still growing until finally the entire project was cancelled.

The debate about small science versus large science has a special personal significance for me. In 1995 I was a graduate student at the University of Chicago, where I had the honour to serve as Enrico Fermi's assistant for his last course. A few months later Fermi called a meeting of all the students who passed the latest Ph.D. qualifying exam, among whom I was included. He told us that although the first post-war cyclotron, which was built at the University of Chicago in his honour, was operating well, the field of high-energy physics was overcrowded. He strongly recommended that we consider many other interesting fields of physics, including several that he had researched, such as atomic and solid-state physics. More recently, the last letter I received from Linus Pauling before his death last year indicated his strong support for a letter I had written to Physics Today criticizing large science in general and the SSC and the NASA space programme in particular.

The great advantage of small science is that it encourages individual initiative. The way this occurs in solid-state physics was dramatized for me by a year of postdoctoral study in 1959 at the University of California at Berkeley. Charles Kittel had already written the first edition of his famous Introduction to Solid-State Physics. The breadth of his knowledge gave him the special ability to direct his students' research in the context of four or five small problems, rather than one large one, for each student. Many of his students became famous, and I was not the only young theorist Kittel attracted to Berkeley as I overlapped with John Hubbard and Pierre de Gennes.

Even the method of many small problems rather than one large one, has its limitations, and my model for guiding student research today is provided by Alex Zettl and Marvin Cohen. They are at Berkeley, but they have refined and formalized the procedures used by Kittel with a number of experimental colleagues in the 1950 s and 1960s. Today Zettl and Cohen have separate team (group) meetings with their students each week, where they discuss fullerene geometries. Marvin Cohen has told me that the most creative and most successful joint meetings have been the ones at which one or both teams have nothing new to report. Formal presentations, it seems, have a deadening affect on the creation of new ideas.

It can be argued that large scale has its team meetings, where attention is focused on a common goal - a large accelerator experiment, or a new rocket mission, for example. But this is just the problem. Such engineering situations, with their long time spans involving many individuals, do not create conditions favourable for individual development. They are necessary in industry, but at the same time they are probably the primary factor responsible for professional obsolescence. We are familiar with the resulting syndrome of technical narrowness, and it is just this syndrome which an education in science is supposed to avoid. Large science reproduces itself at alarmingly rapid rates because of this narrowness, for its protégés have little appeal outside their original research area.

In recent years the appetite of large science has grown most rapidly at a time when resources have been shrinking. It has not always been easy for government agencies to appreciate the differences between large and small science, especially as it is much easier for large science to devote a large fraction of its resources to politics and publicity. Today when resources are so limited these distinctions must be made. Experience has shown overwhelmingly that small is much more successful than large science in developing adaptable and flexible individuals, and these are just the qualities that are valued most highly in industry. That is why from a North American perspective it is surprising that such a large fraction of Europe's resources is still being devoted to large science projects which have so few prospects of educating scientists in areas of economic value to the outside community. 
What little is left of industrial research is still often done the old-fashioned way, but development and production are dominated by team meetings. From the industrial viewpoint, research carried out on a single large problem by one student guided by a professor has several drawbacks. Such research is too narrow, too specific, too deep and, above all, too slow to be of industrial value. The industrial team wants answers in weeks, not years. It much prefers and "on-time" answers, however makeshift, obtained in a few weeks, to a deeper, more elegant answer obtained in several years which comes much too late to help the team. Even the excellent answers are usually to a wrong question.

The most important point to realize is that most industrial practice consists not of deep inventions of carefully prepared discoveries, but of quick and timely incremental steps which either enable new methods or control quality to stabilize and improve the yield of older makeshift methods. Far more money, in general, is made by quality control, for example, than by enabling methods, and far more money is made by the latter for companies than is made for inventions. Once in a while a clever invention saves a company, a classic example being Hewlett-Packard's laser-jet printer. However, clever people alone seldom produce such inventions, and few businesses survive if they devote more than $1 \%$ of their revenues to looking for such inventions in very general terms.

Solid-state physics fits very well into this industrial picture, if it is viewed as providing a basis for students' education which is both technical (by developing expertise in instrumentation) and conceptual (by providing the basis for understanding the properties of materials). Similar skills with regard to instrumentation are developed in electrical engineering, but electrical engineers generally treat material properties as God-given, although in the case of silicon integrated circuits, this approach is well justified. In any case, it is clear that the needs of industry differ greatly from the goals of most traditional academic research.

\section{Go Beyond Fundamentals}

My second thesis concerns the impact of global economics on industrial research. During the last three decades industrial research in solid-state physics has undergone profound changes. General or undirected research at many laboratories has been replaced by research focused on specific products with specific pur- poses. The corresponding markets are called niches, and companies that have been successful in finding niches have done well without the benefit of general research. Not all companies were successful in restructuring their research, and those that failed have largely disappeared. The fate of companies currently undergoing restructuring, such as IBM and AT\&T, remains uncertain. Moreover, even companies which have successfully restructured their research, development and production, such as Xerox and General Electric, still face intense competition in international markets.

This North American picture is part of a much larger picture, which is the continuing industrialization of formerly less industrialized countries, especially in Asia. A second wave of American industrialization began in 1950 in what has come to be called Silicon Valley, and a third wave is emerging there now, based not only on electronics, but on optical fibres and large band-width communications.

All of this high-technology revolution has largely by-passed Europe with its high labour costs, and at present among European companies only Siemens is mounting a large effort in semiconductors. It has been realized for some time that part of the reason for European inactivity was the lack of a common market, and progress has occurred and is still occurring in this political direction. The European Physical Society itself is a part of this larger effort.

The problems that European nations, and to a considerable extent other advanced industrial, face are not, however, primarily political. Manufacturing costs are reduced by cheap labour, but high technology manufacturing cannot be carried out with only cheap labour. In fact, most high-technology manufacturing is automated for better quality control. This requires high-quality engineers. But even with an abundant supply of engineers the generation of new products often requires knowledge at the fundamental level. This, hopefully, is where physicists can make their contributions, but in practice these successes have been less frequent in recent years.

The reason why early successes have not continued lies primarily in the culture of science. The success of science in generating new products has dazzled not only the general public, but also (in the past) many business managers as well. However, the population of scientists and engineers has grown more rapidly than the world's population owing to fostering by govern- ment subsidies associated with the cold war. Moreover, many scientists have retained their academic cultural orientation throughout their careers so highquality scientific research has tended to be less commercially oriented. This has made it less economically rewarding to pursue such research in industry: whatever you need in general knowledge probably already exists in the literature, thus reducing the need for industrial research.

The evolution of industrial solid-state physics in Japan since 1950 has been both rapid and instructive. Initially Japan benefited from a highly skilled and very cheap domestic labour supply. This enabled Japan to dominate the world consumer electronics and high-quality automobile markets. Success, especially in consumer electronics, was made possible by the existence of a large number of scientists and engineers who were well educated in solidstate physics. Today Japan's labour is much more expensive, and labour costs in Japan are comparable to those in Germany and the US. New factories are being placed often outside Japan, not only in areas of cheap labour, but for political reasons in Europe and the US as well. Once again the location of these factories is determined to a considerable extent by the availability of highly skilled and well-educated labour. Solid-state physics is a large part of that education.

The revolutionary changes we are discussing sometimes puzzle physicists as they often feel that they are so strong in matters of general principles and depth of understanding that the world should value them for that alone. But specific industries and even specific nations, cannot achieve prosperity in the highly competitive markets of an overpopulated world simply by consulting physicists who understand general principles. Physicists must be eager to take their understanding and use it to develop specific needs. Again a good example is the laser jet printer, which is not only cheaper, faster and more reliable than old mechanical printers, but also much more pleasant company since it is silent

Inventing a new product is very difficult, but understanding the needs of a niche market and using technical skills based on a good understanding of solidstate physics can be done much more easily. Identifying the niche market and exploiting it is also something which we associate more often with engineers than with physicists, but there is no reason why this should be the case. Although it is not 
part of my business, the telecommunications one, there have been many examples of biomedical instrumentation which have been developed by physicists. I have in mind not only the glamourous and expensive ones (like NMR imaging), but also the many small and simple instruments which have exploited the possibilities of optical fibres, for example. Such instruments can be developed without expensive subsidies in modestly equipped laboratories. Here small teams are needed, much smaller than the teams which developed semiconductor technology, but still the principle of teamwork remains the same.

\section{Disciplined Interactions}

Academics do not fit easily into this brave new world. Teachers at all levels have very broad responsibilities associated with being surrogate parents to hundreds of young people. Most of these young people are not going to be involved in manufacturing, even though the level of manufacturing determines the overall level of prosperity of each country. Some economists have suggested that in 20 or 30 years a kind of equilibrium will develop in manufacturing capacities around the world, as the economies of countries that are now underdeveloped mature. In such a technologically equilibrated world only $10 \%$ of each country's population would be engaged in manufacturing. For presently industrialized countries, this picture has drastic implications. In the US at present about $15 \%$ of our population is engaged in manufacturing, so that if this picture is correct, the fraction will drop by $1 / 3$. For Germany, where the current figure is $32 \%$, the implications are even more drastic. There the fraction could drop by $70 \%$.

We are thus faced with a paradox which is of great importance to scientists. On the one hand each country's prosperity will depend on success in manufacturing. However, if only $10 \%$ of revenues comes from this sector, and more than half of the population obtains advanced education of some kind, then scientists will be spending 80 or $90 \%$ of their time teaching basic science to students who will have no direct applications of this knowledge. Somehow these two points of view must eventually be reconciled.

There is no automatic means or recipe for reconciling manufacturing and educational needs. More than ever, responsible and active government involvement is needed to bring the two cultures together. On the whole Japan seems to have achieved the greatest degree of success in this area. It has generally relied on joint consultations between representatives from universities, industries and government topic selection in a way that is academically feasible and industrially rewarding. This requires, however, a great deal of discipline among academics. In the United States we have not had much of this, as the SSC demonstrated.

The problem of disciplined academicindustrial-governmental interactions is exacerbated by the fact that one school of thought argues that education in applied science should not or cannot be carried out in universities, but that such training should be done in industry itself. My own feeling is that most of the industrial efforts at mentoring which I have observed have been amateurish to the point of absurdity. Graduate schools perform invaluable services by training large groups of young scientists and grading them according to abilities and interests - tasks which cannot be carried out in industry. I have witnessed some of the disasters that can occur when industrial mentors are overcome by virgin pedagogical enthusiasm and try to train technicians to do the jobs of scien- tists. For a very few individual cases successes are possible, but those exceptions are rare.

When we talk of disciplined academic - industrial - governmental relations we mean just that. I believe that much of the disappointment with the results of government-supported research stems primarily from absence of this discipline. It is true that academics guard their independence fiercely, but then no one forces them to accept government funding. It is very tempting to simply divide available funds equally, or give them to interest groups which are largest and can afford to dedicate the largest amount of time to lobbying. This is why large science has received so much in the past. But if one simply asks how many people in industry are doing similar work, and makes a first-order correction for growth, most of these problems can be avoided. It is not too hard to recognize Gresham's law in action, and to identify fields where the pressure is greatest to create government-supported positions for recent graduates who would otherwise have great difficulty finding employment.

\section{Europe Needs Special Measures}

\section{J. Bessa Sousa from the University of Porto argues that the European situation must be placed in the correct context. \\ J. Bessa Sousa}

In spite of Europe's decline in external competitiveness, it has (surprisingly) maintained social welfare growth for many years. But alarming signals are emerging nowadays, notably a large unemployment rate, almost stagnant economies and increasing difficulties in the process of European integration. High salaries combined with low rates of industrial production and innovation are different views of the same problem.

It will be difficult to improve consistently the quality and cultural aspects of life in Europe without a major internal restructuring process, for economic integration alone seems insufficient to promote the necessary global transformation. When planning for the 21st century, Europe should select the most promis ing emerging areas of science and technology and promote priority research and concerted actions in them. But it will be erroneous to assume that this will be enough to put Europe in the front row insofar as the competitive exploitation of new technologies.

A critical assessment should be made of the actual strengths and limitations of Europe's science and technology before embarking on a concerted strategic plan for the future. The present shortcomings and limitations, if not properly corrected, will constitute a very shaky basis for implementing any strategic plan.

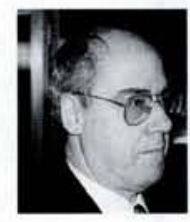

For example, science and technology may not be adequately mature in Europe as a whole. In other words, they may not be adequately developed and implemented in the various regions and countries. For one can ask whether Europe is taking full advantage of all of its human resources and research capacities, and indeed whether it can compete with scientifically more homogeneous and integrated countries such as Japan or USA.

At present, only a few European countries participate in true sense in meaningful international competition, and only a very few companies are involved. Europe must promote a more balanced participation, with an improved distribution of its scientific and technological expertise, and of the benefits resulting from the industrial applications. Without a more participative global effort it will be difficult to cope with the competition (and faster development) coming from Asia and North America.

Therefore, besides economic integration Europe needs an integration programme in science, technology and industry aimed at a more homogeneous distribution of medium-size research facilities and of technological expertise and skills, and the creation of a real partnership in developing and setting up the new industries which are needed. This programme should also lead to a proper integration of the 